\title{
SPINK1 promotes cell growth and metastasis of lung adenocarcinoma and acts as a novel prognostic biomarker
}

\author{
Liyun Xu ${ }^{1}$, Changchang $\mathrm{Lu}^{1}$, Yanyan Huang ${ }^{1}$, Jihang Zhou ${ }^{1}$, Xincheng Wang ${ }^{1}$, Chaowu Liu ${ }^{2}$, Jun Chen ${ }^{2} \mathcal{E}$ Hanbo Le $^{2, *}$ \\ ${ }^{1}$ Cell and Molecular Biology Laboratory, Affiliated Zhoushan Hospital of Wenzhou Medical University, Zhoushan 316000, ${ }^{2}$ Department of \\ Cardio-Thoracic Surgery, Affiliated Zhoushan Hospital of Wenzhou Medical University, Zhoushan 316000, China
}

\begin{abstract}
Serine protease inhibitor Kazal type 1 (SPINK1) plays a role in protecting the pancreas against premature activation of trypsinogen and is involved in cancer progression. SPINK1 promoted LAC cells growth, migration, and invasion. Mechanistically, we found that SPINK1 promoted LAC cells migration and invasion via up-regulating matrix metalloproteinase 12 (MMP12). We observed that SPINK1 expression was only up-regulated in lung adenocarcinoma (LAC) tissues, and was an independent prognostic factor for poor survival. Our results indicate that SPINK1 might be a potential biomarker for LAC that promotes progression by MMP12. [BMB Reports 2018; 51(12): 648-653]
\end{abstract}

\section{INTRODUCTION}

Lung cancer is one of the most common malignant tumors, and has become the leading cause of cancer deaths in both males and females worldwide (1). Non-small-cell lung cancer (NSCLC) accounts for about $80 \%$ of all diagnosed lung cancer, and lung adenocarcinoma (LAC) is the most common histologic subtype of NSCLC (2). Despite the advancement of targeted therapy with significant responses, LAC treatment remains a challenge because of acquired resistance and unknown driver factors (3). Therefore, there exists a need to identify new molecular regulators for LAC, providing insights into potential therapeutic targets.

The serine protease inhibitor Kazal type 1 (SPINK1) gene, also known as pancreatic secretory trypsin inhibitor (PSTI) or tumor associated trypsin inhibitor (TATI), encodes a 79 amino acid peptide, and mature SPINK1 consists of 56 amino acids $(4,5)$. The physiological function of SPINK1 is to inhibit the

*Corresponding author. Tel: +86-580-2292772; Fax: +86-580-229 2518; E-mail: zslehanbo@163.com

https://doi.org/10.5483/BMBRep.2018.51.12.205

Received 4 September 2018, Revised 22 October 2018, Accepted 15 November 2018

Keywords: Biomarker, Cell growth, Lung adenocarcinoma, Metastasis, SPINK1 activation of trypsins in the pancreas, and prevent "self-digestion" of the organ (6). In the analysis of structure, SPINK1 shares $50 \%$ homology with epidermal growth factor (EGF) in the sequences of amino acid, and can bind with epidermal growth factor receptor (EGFR) (7). Therefore, it was inferred that SPINK1 plays a growth factor role. Recent studies have proved that SPINK1 can promotetumor cell growth in prostate (8), colon (9, 10), pancreatic (11) and ovarian cancer (12), which indicated that SPINK1 has a protumorigenic role through a mechanism distinct from its classical activity as a serine protease inhibitor. Previous studies suggested the potential role of SPINK1 in tumor development and progression, and that it might represent a useful therapeutic target for cancer. However, its expression pattern and biological functions in LAC remain unclear.

In our study, SPINK1 promoted LAC cell growth, migration and invasion. Furthermore, we found that SPINK1 promoted LAC cells migration and invasion via up-regulating matrix metalloproteinase 12 (MMP12). Importantly, we showed that SPINK1 over-expression correlated with poor prognosis of LAC patients.

\section{RESULTS}

Down-regulation of SPINK1 attenuates LAC cells growth and metastasis

To explore the differentially expressed genes (DEGs) in LAC tissues, microarray was performed on LAC patient tissues (Supplementary Table S1). The heat map diagram shows the result of a two-way hierarchical clustering based on the 50 most up-regulated DEGs between LAC tissues and normal lung tissues (Supplementary Fig. S1A). The top 10 significantly up-regulated DEGs are listed in Supplementary Table S2 and ranked by fold-change, compared to normal tissues. We found only SPINK1 mRNA was significantly up-regulated in LAC tissues (Supplementary Fig. S1B).

To explore the role of SPINK1 in LAC development, we first evaluated the SPINK1 expression levels in LAC cell lines and found that SPINK1 mRNA was higher in $\mathrm{H} 1975$ and A549 cells, and lower in H1299 and PC9 cells (Fig. 1A). SPINK1 siRNAs (siRNA 1, siRNA 2 and siRNA 3) were transfected into LAC cell lines (A549 and H1975), and it was confirmed that

ISSN: 1976-670X (electronic edition)

Copyright (C) 2018 by the The Korean Society for Biochemistry and Molecular Biology

c) This is an open-access article distributed under the terms of the Creative Commons Attribution Non-Commercial License (http://creativecommons.org/licenses/by-nc/4.0) which permits unrestricted non-commercial use, distribution, and reproduction in any medium, provided the original work is properly cited. 


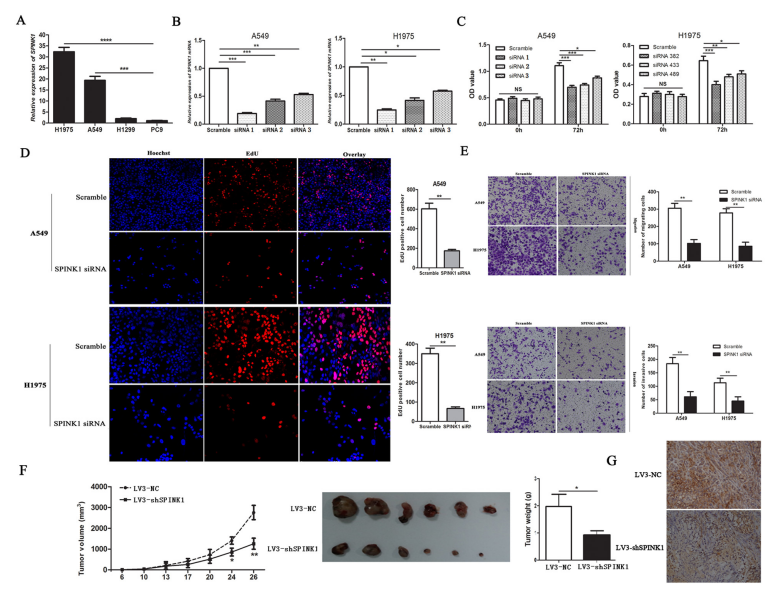

Fig. 1. SPINK1 inhibition suppresses the growth, migration and invasion of LAC cells. (A) SPINK1 mRNA levels were detected in LAC cell lines by qRT-PCR normalized to GAPDH. (B) Detection of the interference effect of SPINK1 siRNAs in LAC cell lines by qRT-PCR. (C) At $0 \mathrm{~h}$ and $72 \mathrm{~h}$, cell growth analysis of A549 and H1975 cells transfected with SPINK1 siRNAs or scramble siRNA by CCK-8 assay. (D) At $72 \mathrm{~h}$, cell proliferation was assayed by EdU. Scale bars, $100 \mu \mathrm{M}$. (E) The migration and invasion ability were assessed by the Transwell assay. Scale bars, $100 \mu \mathrm{M}$. (F) SPINK1- knockdown (LV3-shSPINK1) A549 cells $\left(5 \times 10^{6}\right)$ or the control (LV3-NC) in $200 \mu \mathrm{l}$ PBS were subcutaneously injected into the left axillae of the BALB/C nude mice. The tumor size was monitored 2 times weekly. Tumor volume was calculated using the formula: $V=$ length $\times$ width $\times$ width/2. and a growth curve was drawn. After 26 days, the mice were euthanized, and the tumors were isolated and weighed. (G) The SPINK1 expression in xenograft tissues was measured by IHC $(200 \times)$. *P $<0.05$, **P $<0.01, * * * P<0.001$.

SPINK1 expressions were successfully down-regulated (Fig. 1B). As shown in Fig. 1C, SPINK1 inhibition significantly reduced the growth of $\mathrm{A} 549$ and $\mathrm{H} 1975$ cells. The EdU immunofluorescence staining showed that EdU-positive cells were reduced, indicating suppression of SPINK1, in both A549 and $\mathrm{H} 1975$ cells (Fig. 1D). Further, we explored the effects of SPINK1 on the migration and invasion of LAC cells using the transwell system. Representative micrographs of transwell filters can be seen in Fig. 2E. The migrating and invasive potential of LAC cells were markedly weakened in SPINK1 siRNA (siRNA 1) group, compared to the scramble group (Fig. $1 \mathrm{E}, \mathrm{P}<0.01)$. Additionally, the A549 xenografts displayed significant inhibition of tumor growth in the group of LV3-shSPINK1, compared to LV3-NC (Fig. 1F). Furthermore, a significant reduction in SPINK1 expression was observed in tumor tissues from LV3-shSPINK1 group, compared to LV3-NC (Fig. 1G, Supplementary Fig. S2A). These results proved that SPINK1 inhibition suppressed the development of lung adenocarcinoma.

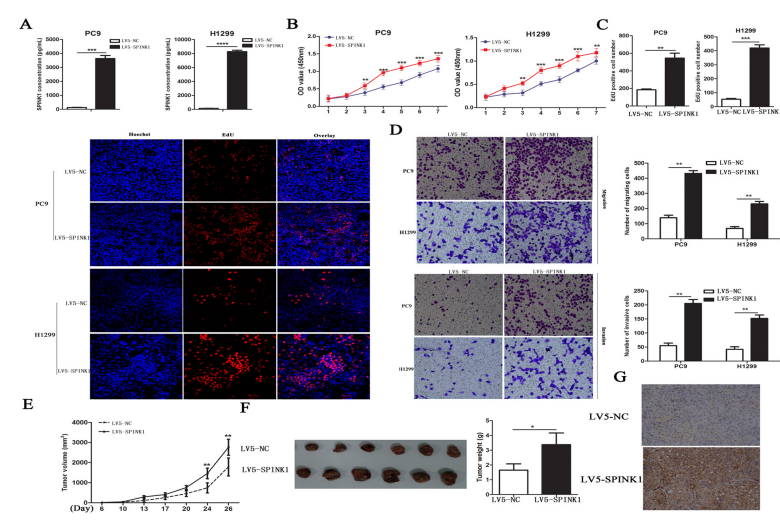

Fig. 2. SPINK1 overexpression promotes the growth, migration and invasion of LAC cells. (A) SPINK1 protein level was detected by ELISA in cultures of stable SPINK1-overexpressing PC9 and H1299 cells. (B) The cell growth was monitored by CCK-8 assay for 7 days. (C) Cell proliferation was assayed by EdU staining. Scale bars, $100 \mu \mathrm{M}$. (D) The migration and invasion ability were assessed by the Transwell assay. Scale bars, $100 \mu \mathrm{M}$. (E) PC9-LV5-control or PC9-LV5-SPINK1 cells $\left(5 \times 10^{6}\right)$ in $200 \mu \mathrm{l}$ PBS were Subcutaneously injected into the left axillae of the $\mathrm{BALB} / \mathrm{C}$ nude mice. The tumor size was monitored 2 times weekly. Tumor volume was calculated using the above formula and growth curve was drawn. (F) After 26 days, the mice were euthanized, and the tumors were isolated, imaged and weighed. (G) The SPINK1 expression in xenograft tissues was measured by IHC $(200 \times)$. *P $<0.05$, **P $<0.01$, ***P $<0.001$.

\section{SPINK1 up-regulation promotes LAC cell proliferation, migration, and invasion}

To further explore the effect of SPINK1 on LAC development, LAC cells (PC9, H1299) with very low SPINK1 expression levels were infected with LV5-SPINK1 or LV5-NC.

Our results showed that SPINK1 expression was significantly increased in the LV5-SPINK1 group, compared to the LV5-NC group (Fig. 2A). Results also showed that the growth rate of LV5-SPINK1-infected cells was significantly higher than that of the control group (Fig. 2B). Furthermore, we determined that the EdU-positive cells were increased in both PC9 and H1299 cells, in which SPINK1 expression was overexpressed (Fig. 2C). We also examined the effect of SPINK1 on the migrated and invading abilities of LAC cells, and it was found that SPINK1 overexpression promoted the migration and invasion of PC9 and H1299, as shown by the transwell assay (Fig. 2D). To further elucidate the role of SPINK1 overexpression in tumor growth ability in vivo, we established mouse xenograft models with stable SPINK1 overexpressing PC9 cells, or control cells, by subcutaneous injection. SPINK1 overexpression significantly promoted tumor formation and tumor growth (Fig. $2 \mathrm{E}$ and $2 \mathrm{~F}$ ). SPINK1 expression was up-regulated in tumor tissues from the LV5-SPINK1 group, compared with the LV5-NC group (Fig. 2G, Supplementary Fig. S2B). These results demonstrated that SPINK1 played a pro-tumor role by promoting cell proliferation and invasion. 


\section{SPINK1 facilitates migration and invasion in LAC cells via up-regulating MMP-12}

Matrix metalloproteinases (MPPs) play important roles in tumor invasion and metastasis. Thus, we investigated whether SPINK1 was involved in regulating MMPs expressions in LAC cells. As shown in Fig. 3A, MMP12 mRNA expression was down-regulated, while other mRNAs were not significantly down-regulated in A549 and $\mathrm{H} 1975$ cells. We also confirmed that MMP12 protein was decreased in SPINK1 knockdown cells (Fig. 3B). Compared to the control, MMP12 protein levels were up-regulated in SPINK1-overexpressing PC9 and H1299 cells (Fig. 3C). Next, we examined the MMP12 expression of tumor tissues in xenograft models with LAC cells, in which SPINK1 expressions were knocked-down or over-expressed. As shown in Fig. 3D, MMP12 mRNA was down-regulated the of SPINK1 knockdown group and up-regulated in the SPINK1 overexpression group. Furthermore, the level of SPINK1 mRNA expression was significantly positively correlated with the level ofMMP12 mRNA in LAC tissues $(\mathrm{R}=0.709, \mathrm{P}<$ 0.001) (Fig. 3E). To further elucidate the effects of SPINK1 on LAC cells migration and invasion, dependent on MMP12, MMP12 was knocked down by siRNA (Fig. 3F). The results showed that the enhanced migratory and invasion ability of

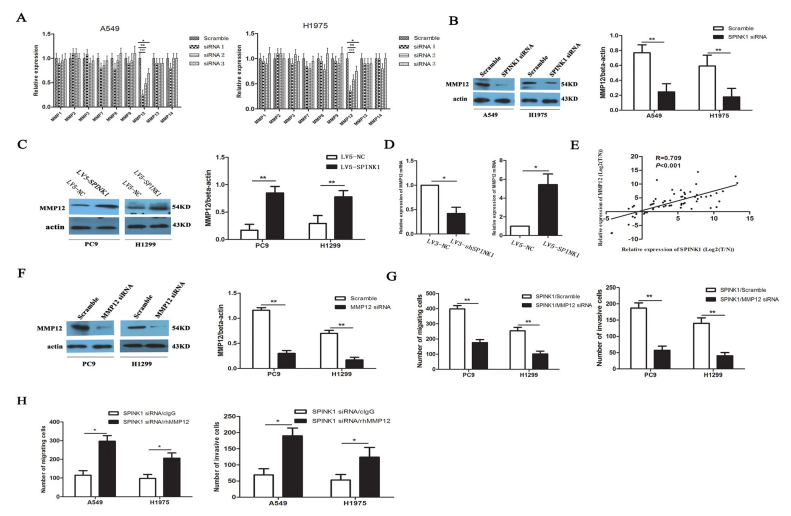

Fig. 3. SPINK1 enhances the migration and invasion of LAC cells by up-regulating MMP12. (A) MMPs expressions were analyzed by qRT-PCR in A549 cells and H1975 cells transfected with scramble or SPINK1 siRNAs. (B) MMP12 protein expressions were analyzed by Western blot in A549 cells and H1975 cells transfected with scramble or SPINK1 siRNA 1. (C) MMP12 protein levels were measured by Western blot in PC9 cells and $\mathrm{H} 1299$ cells transfected with control or SPINK1. (D) MMP12 mRNA expression in xenograft tissues was measured by qPCR. (E) The correlation between SPINK1 expression and MMP12 expression in LAC tissues was analyzed with Pearson correlation. (F) The interference effect of MMP12 siRNA was verified by Western blot in PC9 and H1299 cells stably overexpressing SPINK1. The number of migrating and invasive cells was counted $(\mathrm{G})$. (H) Recombinant human MMP12 (rhMMP12) or control IgG (clgG) at a concentration of $50 \mathrm{ng} / \mathrm{ml}$ was added back into SPINK1 knockdown A549 and H1299 cells, and the number of migrating and invasive cells was counted. ${ }^{* P}$ $<0.05, * * P<0.01, * * * P<0.001$.
SPINK1-overexpressing LAC cells was dramatically reversed (Fig. 3G). Moreover, when recombinant human MMP12 was added back to SPINK1-knockdown A549 and H1975 cells, the numbers of migrating and invasive cells were rescued (Fig. $3 \mathrm{H})$. Collectively, SPINK1 promoted the migration and invasion of lung adenocarcinoma by up-regulating MMP12 expression.

\section{Increased SPINK1 expression in LAC tissues significantly correlates with poor prognosis}

Since SPINK1 can promote LAC cell growth and metastasis, we next explored thecorrelation between SPINK1 expression and prognosis in LAC patients. First, wedetected SPINK1 expression in tissues with LACs, lung squamous cell carcinoma (Sq), or nonmalignant lung nodules (NLNs), and our results showed that SPINK1 protein was only up-regulated in LAC tissues (Fig. 4A, Supplementary Fig. S3 and Table S3). Second, we explored the correlation between SPINK1 expressionand clinicopathological features. The results indicated that SPINK1 expression was significantly positively associated with male gender, carcinoembryonic antigen (CEA) level, tumor size, invasive histology (Fig. 4B), lymph node metastasis, and advanced stage disease (Supplementary Table S4). More importantly, higher expression of SPINK1 in LAC tissues was negatively correlated with progression-free survival (PFS) and overall survival (OS) in the cohort of patients (Fig. 4C). Furthermore, a Cox proportional hazards model showed

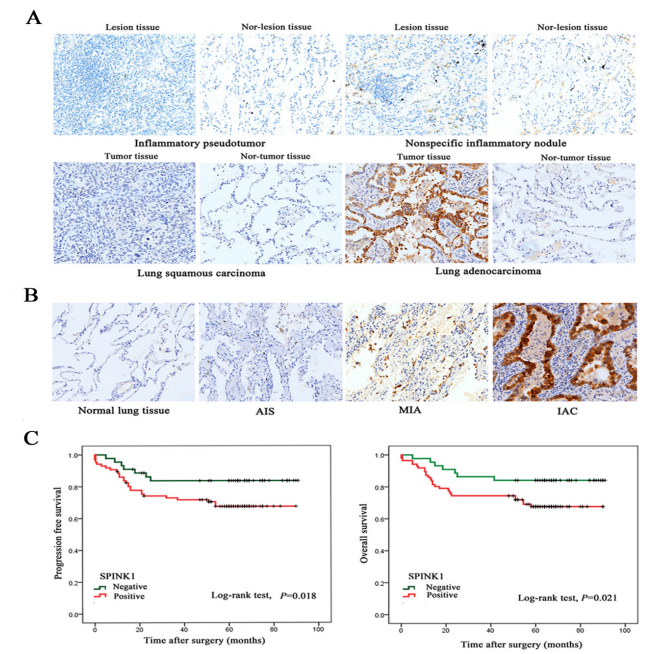

Fig. 4. Enhanced SPINK1 expression in LAC predicts poor prognosis. (A) Representative images of IHC staining of SPINK1 protein in NLNs, Sq, LAC, and matched non-tumor tissues $(\times 200)$. (B) SPINK1 expressions in subtypes of LAC. Representative images of IHC staining of SPINK1 protein in AIS, MIA, IAC and nor-tumor tissues $(\times 200)$. (C) The rate of PFS and OS of LAC patients with higher SPINK1 expression was significantly lower than that of the patients with lower SPINK1 expression. $* \mathrm{P}<0.05,{ }^{*} \mathrm{P}<0.01,{ }^{* * *} \mathrm{P}<0.001$. 
that SPINK1 remained significantly associated with PFS and OS. Supplementary Table S5 showed the results of the univariate and multivariate analyses. Taken together, our results suggested that SPINK1 might act as a novel prognostic biomarker for LAC.

\section{DISCUSSION}

Several studies have reported that SPINK1 stimulated growth of several cancers, including pancreatic cancer (13), prostate cancer (14), ovarian cancer (12), and colorectal cancer (15), suggesting that SPINK1 may significantly promote cancer cell growth and progression. In our study, we found that knockdown of SPINK1 decreased the proliferative ability of LAC cells, whereas overexpression of SPINK1 had the opposite effect. Our findings can be explained by the fact that the structure of SPINK1 is similar to EGF (7), and can stimulate cell proliferation by the EGFR/MAPK signaling pathway (16). The results of our current study are consistent with those of previous studies and indicate that SPINK1 plays an important role in the promoting the growth of LAC cells, which could offer a new target for pharmacological intervention in LAC patients with SPINK1-expressing tumors.

Tumor metastasis, seen in more than $90 \%$ of all tumors deaths, is considered to be the leading cause of mortality in cancer patients, but the underlying mechanisms remain unclear. SPINK1 has been reported to be related to tumor metastasis $(17,18)$. Our data showed that SPINK1 expression levels in tissues of invasive adenocarcinoma (IAC) were higher than that of minimally invasive adenocarcinoma (MIA) adenocarcinoma in situ (AIS), and increased expression of SPINK1 was closely correlated with lymph node metastases, strongly suggesting that SPINK1 may be involved in the LAC metastatic processes. In this study, knockdown of SPINK1 can inhibit the migration and invasion of LAC cells, whereas SPINK1 overexpression can promote this effect. The results can be explained by the finding that SPINK1 can promote the process of epithelial-mesenchymal transition (EMT) (19). EMT is an important biological process that allows malignant tumor cells to acquire migration and invasion capabilities. Furthermore, we explored the underlying mechanisms responsible for SPINK1 promoting-metastasis in LAC. MMPs are known to degrade almost all protein components in the extracellular matrix and play a key role in the invasion and metastasis of tumors. Many studies have confirmed that the MAPK signaling pathway can regulate the expression of MMPs, thereby affecting the migration and invasion of tumors $(20,21)$. Pornchai et al. found that ligands of EGFR could regulate MMP9 to different degrees in head and neck cancer (22), suggesting that SPINK1, as an EGFR ligand, could regulate the expression of MMPs. Therefore, we detected the expressions of MMPs family members in SPINK1-modifed LAC cells and found that only MMP12 expression was dysregulated, which was also proven by in vivo experiments. Moreover, it was confirmed that SPINK1 expression was positively correlated with MMP12 expression in LAC clinical samples. All of these data suggested that MMP12 might be an important mediator in SPINK1-regulated invasiveness and metastasis of LAC cells. Subsequently, we used MMP12 siRNA and rhMMP12 to further confirm MMP12 mediated the metastatic potential of SPINK1 in LAC cells. Previous studies have demonstrated that MMP12 was involved in the invasion of LAC cells (23), which further supported SPINK1 enhancement of the metastasis of LAC cells via MMP12. Taken together, our findings shed new light on the role of SPINK1 in the metastasis of LAC.

SPINK1 was overexpressed and correlated with prognosis in a variety of tumors $(12,24-26)$. In our study, it was confirmed that SPINK1 was expressed only in LAC tissues, but not in tissues of Sq and NLNs. In the physical condition, SPINK1 is secreted from pancreatic acinar cells. Lung adenocarcinoma is named for its glandular cavity structure, which is similar to the structure of pancreas. This may be the reason SPINK1 is expressed only in adenocarcinoma. Furthermore, our study showed that higher SPINK1 levels in tumor tissues was correlated with shorter PFS and OS in LAC patients. This can be explained by our finding that SPINK1 can promote LAC cells growth. Thus, we present very important clinical evidence, suggesting that SPINK1 might serve as a novel prognostic biomarker for LAC and is also involved in LAC progression.

In summary, we initially demonstrated the effect of SPINK1-promoting growth and the underlying molecular mechanisms that SPINK1 promotes metastasis of lung adenocarcinoma by MMP12. Importantly, our results suggested that SPINK1 might be a potential biomarker for lung adenocarcinoma. Our findings provide new insight into the lung adenocarcinoma pathogenesis mediated by SPINK1, and a novel target candidate for effective lung adenocarcinoma therapy.

\section{MATERIALS AND METHODS}

\section{Patients and tumors}

For mRNA array analysis, tumor and paired adjacent non-tumor tissues from 6 LAC patients were used (Supplementary Fig. S4). For qRT-PCR analysis, we used fresh tissues of 62 primary LACs. For immunohistochemical analysis, primary tumor and paired adjacent nontumor tissues were collected from 382 LACs, $45 \mathrm{Sq}$, and 51 NLNs, from patients who underwent surgical resection between January 2010 and June 2013, at the Department of Cardiothoracic Surgery of Zhoushan hospital. No patients had received chemotherapy or radiation before resection. Informed consent was obtained from all patients before the study was initiated with approval of the Zhoushan Hospital Ethics Committee in accordance with the Declaration of Helsinki. 


\section{Gene expression analysis by microarray}

To accurately acquire tumor cells and normal cells from lung tissues, samples were microdissected using a laser capture microdissection system (Applied Biosystems ${ }^{\mathrm{R}}$ Arcturus XT $^{\mathrm{TM}}$, $A B I)$. mRNA profiling was performed using Illuminia Technologies Human Genome U133 Plus 2.0 Array, according to the manufacturer's protocol. GenomeStudio 1.0 was used to perform average normalization of the results from the mRNA microarray. Microarray data were deposited in a Gene Expression Omnibus (GEO) database (accession no. GSE118370).

\section{RNA extraction and quantitative real-time PCR (qRT-PCR)} Isolation of total RNA and synthesis of CDNA were made according to the manufacture's protocol. qRT-PCR was performed with SYBR Green Realtime PCR Master Mix (Applied Biosystems), and was run on ABI 7500 Real-time PCR system (Thermo, Waltham, MA) . The target gene $\mathrm{Ct}$ values were normalized to GAPDH using the $2-\Delta \Delta \mathrm{Ct}$ method. Gene-specific primers were listed in Supplementary Table S6.

\section{Cell lines, siRNA, Recombinant protein and Plasmids}

The human LAC cell lines NCl-H1975, A549, NCl-H1299 and PC9 were purchased from the Shanghai Cell Collection. The siRNAs were purchased from Life Technologies Corporation (Thermo Fisher Scientific). The SPINK1 siRNAs were used: siRNA1 (HSS144065), siRNA 2 (HSS144065), siRNA 3 (HSS186064), and SilencerTM Negative Control \#1 siRNA (AM 4611). The ID of MMP12 siRNA (AM16708) was 104022 and the control catalog number was AM 4613. Recombinant human MMP12 (rhMMP12, 917-MPB) and the control IgG (clgG, 1-001-A) were purchased from R\&D Systems. Human SPINK1-expressing plasmids pcDNA3.0-SPINK1 (pcSPINK1), and the control plasmid pcDNA3.0 were purchased from GenePharma (Shanghai, China).

\section{Generation of stable cells using lentiviral infection}

To generate stable SPINK1 knockdown cells, A549 and H1975 cells were infected with LV3-pGLV-SPINK1 shRNA lentivirus (LV3-shSPINK1, GenePharma, Shanghai, China). To generate stable SPINK1-overexpressing cells, PC9 and H1299 cells were infected with LV5-EF1a-SPINK1 lentivirus (LV5-SPINK1, GenePharma, Shanghai, China). Cells infected with vectorscramble sequence were used as controls (LV3-NC, LV5-NC). Single-cell clones were isolated by $5 \mu \mathrm{g} / \mathrm{ml}$ Puromycin for $48 \mathrm{~h}$ followed by $1 \mu \mathrm{g} / \mathrm{ml}$ puromycin treatment. SPINK1 expressions were confirmed by qRT-PCR (Supplementary Fig. S5).

\section{Enzyme-linked Immunosorbent Assay (ELISA)}

The concentration of SPINK1 in the cell culture supernatant was determined using the ELISA kit from Abnova (KA4275, Taiwan). The assays were performed according to the manufacturer's instructions. All samples were examined in duplicate.

\section{Cell Counting Kit-8 analysis}

LAC cells were seeded in 96-well cell culture plates. Cell viability was measured using Cell Counting Kit-8 (CCK-8, C008-3, 7 sea biotech, Shanghai, China).

\section{EdU immunofluorescence staining}

Immunofluorescence staining was performed according to standard protocols using Click-iT EdU Imaging Kits (C10338, Invitrogen).

\section{In vitro migration and invasion assays}

Cell migration assays were performed using $6.5-\mathrm{mm}$ transwell chambers ( $8 \mu \mathrm{m}$ pore size, Corning, USA). Cell invasion assays were performed using the Cell Invasion Assay Kit (ECM550, Millipore, USA), according to the manufacturer's protocol.

\section{In vivo tumor homograft assay}

Male BALB/c nude mice (6-8 weeks of age, 16-18 g) were purchased from Vital River Laboratory Animal Technology Co. Ltd (Beijing, China). All mice were housed in the animal facilities under the specific pathogen-free (SPF) conditions. The welfare of experimental mice followed Guide for the Care and Use of Laboratory Animals.

All mice were randomly divided into four groups, and a xenograft model was established by subcutaneous injection of $0.2 \mathrm{ml}\left(5 \times 10^{6}\right)$ A549 cells stable knockdown of SPINK1, PC9 cells stably overexpressing SPINK 1 , and their controls. Tumor size was monitored 2 times weekly for 20-30 days. Tumor volumes $(\mathrm{V})$ were calculated by the equation $(\mathrm{V}=$ length $\times$ width $\times$ width/2). Mice were euthanized, and the tumors were isolated and weighed. Animal experiments were repeated two times and at least six mice were included in each cohort.

\section{Westem blot analysis}

Cells were lysed, and proteins were detected as described previously. Immunoblotting was carried out with the anti-MMP12 (sc133151, Santa Cruz Biotechnology, USA) and anti- $\beta$-actin (TA-09, Zhongshanjinqiao Biotechnology Co., Beijing, China).

\section{Immunohistochemical (IHC) staining}

IHC staining was performed according to standard protocols using the following antibody: anti-SPINK1 (ab183034, Abcam, UK). Positive staining areas in the entire tissue section were graded as follows: 0 , for $5 \%$; 1 , for $5-25 \%$; 2 , for $26-50 \% 3$, $51-100 \%$. Tumors scored as 0 or 1 were considered negative, and those scored as 2 or 3 were classified as positive.

\section{Statistical analysis}

GraphPad Prism 5.0 (GraphPad Software, San Diego, CA, USA) was used for data analysis. P-values of $<0.05$ were considered to be statistically significant. 


\section{ACKNOWLEDGEMENTS}

This work was supported in part by grants from Science Technology Department of Zhejiang Province of China (nos. LGF18H160008, 2015C37024， 2017C37047), Bureau of Science and Technology of Zhoushan (nos. 2014C31065, 2016C13037) and Bureau of Health and family planning of Zhoushan (no. 2017G02).

\section{CONFLICTS OF INTEREST}

The authors have no conflicting interests.

\section{REFERENCES}

1. Paci E, Puliti D, Lopes Pegna A et al (2017) Mortality, survival and incidence rates in the ITALUNG randomized lung cancer screening trial. Thorax 72, 825-831

2. Parkin DM, Bray F, Ferlay J et al (2002) Global cancer statistics. CA Cancer J Clin 55, 74-108

3. Molina JR, Yang P, Cassivi SD et al (2008) Non-small cell lung cancer: epidemiology, risk factors, treatment, and survivorship. Mayo Clin Proc 83, 584-594

4. Stenman UH (2002) Tumor-associated trypsin inhibitor. Clin Chem 48, 1206-1209

5. Itkonen O and Stenman UH (2014) TATI as a biomarker. Clin Chim Acta 431, 260-269

6. Stenman UH, Huhtala ML, Koistinen R et al (1982) Immunochemical demonstration of an ovarian cancer-associated urinary peptide. Int J Cancer 30, 53-57

7. Scheving LA (1983) Primary amino acid sequence similarity between human epidermal growth factorurogastrone, human pancreatic secretory trypsin inhibitor, and members of porcine secretin family. Arch Biochem Biophys 226, 411-413

8. Ateeq B, Tomlins SA, Laxman B et al (2011) Therapeutic targeting of SPINK1-positive prostate cancer. Sci Transl 3, 72ra17

9. Ida S, Ozaki N, Araki K et al (2015) SPINK1 status in colorectal cancer, impact on proliferation, and role in colitis-associated cancer. Mol Cancer Res 13, 1130-1138

10. Tiwari R, Pandey SK, Goel S et al (2015) SPINK1 promotes colorectal cancer progression by downregulating Metallothioneins expression. Oncogenesis 10, 4:e162

11. Zhang J, Wang D, Hu N et al (2016) The construction and proliferative effects of a lentiviral vector capable of stably overexpressing SPINK1 gene in human pancreatic cancer AsPC-1 cell line. Tumour Biol 37, 5847-5855

12. Mehner C, Oberg AL, Kalli KR et al (2015) Serine protease inhibitor Kazal type 1 (SPINK1) drives proliferation and anoikis resistance in a subset of ovarian cancers. Oncotarget 6, 35737-35754

13. Ateeq B, Tomlins SA, Laxman B et al (2011) Therapeutic targeting of SPINK1-positive prostate cancer. Sci TransI Med 3, 72ra17

14. Chen YT, Tsao SC, Yuan SS et al (2015) Serine protease inhibitor Kazal type 1 (SPINK1) promotes proliferation of colorectal cancer through the epidermal growth factor as a prognostic marker. Pathol Oncol Res 21, 1201-1208

15. El-mezayen HA, Metwally FM and Darwish H (2014) A novel discriminant score based on tumor-associated trypsin inhibitor for accurate diagnosis of metastasis in patients with breast cancer. Tumour Biol 35, 2759-2767

16. Ozaki N, Ohmuraya M, Hirota M et al (2009) Serine protease inhibitor Kazal type 1 promotes proliferation of pancreatic cancer cells through the epidermal growth factor receptor. Mol Cancer Res 7, 1572-1581

17. Gaber A, Johansson M, Stenman UH et al (2009) High expression of tumour associated trypsin inhibitor correlates with liver metastasis and poor prognosis in colorectal cancer. Br J Cancer 100, 1540-1548

18. Tomlins SA, Rhodes DR, Yu J et al (2008) The role of SPINK1 in ETS rearrangement-negative prostate cancers. Cancer Cell 13, 519-528

19. Ying HY, Gong CJ, Feng $Y$ et al (2017) Serine protease inhibitor Kazal type 1 (SPINK1) downregulates E-cadherin and induces EMT of hepatoma cells to promote hepatocellular carcinoma metastasis via the MEK/ERK signaling pathway. J Dig Dis 18, 349-358

20. Tania M, Khan MA and Fu J (2014) Epithelial to mesenchymal transition inducing transcription factors and metastatic cancer. Tumour Biol 35, 7335-7342

21. Chakraborti S, Mandal M, Das S et al (2003) Regulation of matrix metalloproteinases: an overview. Mol Cell Biochem 253, 269-285

22. O-charoenrat P, Modjtahedi H, Rhys-Evans P et al (2000) Epidermal growth factor-like ligands differentially up-regulate matrix metalloproteinase 9 in head and neck squamous carcinama cells. Cancer Res 60, 1121-1128

23. Lv FZ, Wang JL, Wu Y et al (2015) Knockdown of MMP12 inhibits the growth and invasion of lung adenocarcinoma cells. Int J Immunopathol Pharmacol 28, 77-84

24. Flavin R, Pettersson A, Hendrickson WK et al (2014) SPINK1 protein expression and prostate cancer progression. Clin Cancer Res 20, 4904-4911

25. Zhang X, Yin X, Shen P et al (2017) The association between SPINK1 and clinical outcomes in patients with prostate cancer: a systematic review and meta-analysis. Onco Targets Ther 10, 3123-3130

26. Räsänen $\mathrm{K}$, Itkonen $\mathrm{O}$, Koistinen $\mathrm{H}$ and Stenman UH (2016) Emerging Roles of SPINK1 in Cancer. Clin Chem $62,449-457$ 